Artículo

\title{
Variabilidad fenotípica de aislados de Phytophthora capsici en Guanajuato
}

\author{
José Luis Pons-Hernández ${ }^{1}$ \\ Brenda Zulema Guerrero-Aguilar ${ }^{1}$ \\ Mario Martin González-Chavira ${ }^{1 \S}$ \\ Enrique González-Pérez ${ }^{1}$ \\ Salvador Villalobos-Reyes ${ }^{1}$ \\ Claudia Ivonne Muñoz-Sánchez ${ }^{2}$ \\ ${ }^{1}$ Centro de Investigación Regional del Centro-INIFAP. Carretera Celaya-San Miguel de Allende km 6.5, \\ Celaya, Guanajuato, México. CP. 38124. (pons.joseluis@inifap.gob.mx; guerrero.brenda@inifap.gob.mx; \\ gonzalez.enrique@inifap.gob.mx; villalobos.salvador@inifap.gob.mx). ${ }^{2}$ Instituto Tecnológico de Celaya. \\ Antonio García Cubas 600, FOVISSSTE, Celaya, Guanajuato, México. CP. 38010. \\ (claudia.munoz@itcelaya.edu.mx). \\ ${ }^{\S}$ Autor para correspondencia: gonzalez.mario@inifap.gob.mx.
}

\section{Resumen}

Phytophthora capsici, es el agente causal de la 'marchitez del chile' una de las enfermedades más importantes de este cultivo. Para establecer estrategias de control eficientes es necesario el conocimiento de la diversidad del patógeno, para esto, se realizó esta investigación cuyo objetivo fue determinar el grado de variabilidad de 29 aislados monozoospóricos obtenidos de chile (Capsicum annuum L.) de seis municipios del estado de Guanajuato y dos cepas de referencia de A1 y A2. Todos fueron caracterizados por la forma de crecimiento, tipo de compatibilidad, crecimiento a $35{ }^{\circ} \mathrm{C}$, sensibilidad a mefenoxam y grado de virulencia. Los resultados obtenidos indican que los aislados, presentaron cinco formas de crecimiento: estrellada $(62.5 \%)$, turuloso (15.62\%), estolonífera (12.5\%), filamentoso $(6.25 \%)$ y petaloide $(3.12 \%)$; su crecimiento a $35{ }^{\circ} \mathrm{C}$ fue menor a $30.7 \%$ respecto al control crecido a $27{ }^{\circ} \mathrm{C}$, su tipo de compatibilidad fue $65.5 \%$ del tipo A1, se encontró sensibilidad intermedia al mefenoxam en $65.5 \%$ de los aislados, siendo el resto sensibles. El aislado más virulento fue el D3 y los avirulentos fueron D8, D11 y D12 todos originarios de Dolores Hidalgo. No se observa una relación directa entre la forma de crecimiento, el crecimiento a $35{ }^{\circ} \mathrm{C}$, el tipo de compatibilidad, la resistencia a mefenoxam y el grado de virulencia.

Palabras clave: diversidad poblacional, marchitez del chile, sensibilidad a mefenoxam.

Recibido: mayo de 2020

Aceptado: septiembre de 2020 


\section{Introducción}

Dentro de los problemas fitosanitarios para la producción del cultivo de chile en México, la 'marchitez del chile' o 'secadera' es la enfermedad más importante, en todas las regiones productoras de chile en el país. En el estado de Guanajuato cerca de 90\% de la superficie sembrada, es afectada en mayor o menor grado por esta enfermedad. En condiciones favorables puede causar pérdidas del 40 al 100\% de la superficie cultivada. La sintomatología de esta enfermedad está asociada con un grupo de organismos siendo el principal Phytophthora capsici Leonian, un oomiceto filamentosos, diploide, heterotálico (Erwin and Ribeiro, 1996).

Se han desarrollado diversas estrategias para el control de éste patógeno, destacando entre ellas: el control químico y cultural, así como el desarrollo de cultivares resistentes. Si se considera que la eficiencia y durabilidad de las medidas de control depende, entre otros factores, de la variación genética del patógeno, sería importante valorarla y conocer su distribución dentro y entre poblaciones de P. capsici, en hospederos o regiones particulares (McDonald y Linde, 2002). La evaluación de la diversidad en la actualidad se basa principalmente en características morfológicas y fisiológicas. Dentro de los caracteres morfológicos destaca el estado sexual de Phytophthora que involucra la producción de dos gametangios morfológicamente diferenciados: el oogonio, estructura femenina A1 y el anteridio, estructura masculina A2 (Martin et al., 2012).

Entre los rasgos fisiológicos más destacados para la caracterización de las especies de Phytophthora son: las temperaturas cardinales de crecimiento, la abundancia de esporulación en medios líquidos, el aspecto de las colonias en medios de cultivo y los engrosamientos hifales. Las colonias de $P$. capsici se caracterizan de acuerdo con el patrón que presentan en medio de cultivo, la ramificación de las hifas puede tener un patrón petaloide, estrellado, en roseta, irregular, concéntrico y con un micelio aéreo ralo, harinoso o algodonoso y sus temperaturas cardinales de crecimiento son: la temperatura mínima $10{ }^{\circ} \mathrm{C}$, la óptima $28{ }^{\circ} \mathrm{C}$ y la máxima para el crecimiento mayor a $35^{\circ} \mathrm{C}$ (Erwin y Ribeiro, 1996).

Otra de las características utilizadas para el estudio de la diversidad y que además tiene implicaciones prácticas, es la resistencia a fungicidas como el metalaxil y el mefenoxam, fungicidas de uso común a nivel mundial para el control de este oomiceto, a los que se han reportado resistencia en poblaciones de todo el mundo (Lamour y Hausbeck, 2003). Los aislamientos resistentes han mostrado ser igual o más virulentos que los sensibles, convirtiendo así la resistencia a estos compuestos en una característica importante para el manejo de esta enfermedad y usada como marcador para caracterizar poblaciones.

\section{Materiales y métodos}

\section{Cepas}

Se utilizaron 29 aislados de $P$. capsici identificados usando las claves de Erwin y Ribeiro (1996), los cuales fueron obtenidos de plantas con síntomas de 'marchitez', en 12 lotes de producción comercial de diferentes variedades e híbridos de chiles tipo poblano, güero, serrano, jalapeño y chilaca, ubicados en los municipios de: Dolores Hidalgo, San Luis de la Paz, Juventino Rosas, Silao, Salvatierra y Cortazar en Guanajuato. 
Las cepas de referencia para los tipos de compatibilidad usadas PCT17 (A1) y PCC6 (A2), fueron donadas por la Dra. Sylvia Patricia Fernández Pavía del Laboratorio de Patología VegetalUMSNH. De todos los aislados incluyendo las cepas de referencia se hicieron cultivos monozoospóricos de acuerdo con el protocolo de Erwin y Ribeiro (1996). Los cultivos se mantuvieron en medio sólido V8C (160 ml de jugo V8, 3 g CaCO 3 y $840 \mathrm{ml}$ de agua desionizada), transfiriéndolos cada seis semanas.

\section{Patrón de crecimiento de la colonia}

Para la descripción del patrón de crecimiento de los aislados se sembraron discos de micelio de 1 $\mathrm{cm}$ de diámetro en agar V8C, se incubaron a $27{ }^{\circ} \mathrm{C}$ durante seis días en la oscuridad. Se determinó las formas de las colonias comparándolas con los patrones descritos por Erwin y Ribeiro (1996).

\section{Crecimiento a $35{ }^{\circ} \mathrm{C}$}

De cada aislado se sembró en el centro de una caja petri con medio V8C, un disco de $8 \mathrm{~mm}$ de diámetro con micelio de 3 a 5 días de edad. Se midió el diámetro de la colonia a lo largo de dos ejes perpendiculares, cada 48 h durante ocho días y la tasa de crecimiento se calculó a 27 y $35{ }^{\circ} \mathrm{C}$. Se realizaron dos ensayos con tres repeticiones para cada aislamiento y se tomó como resultado el promedio obtenido. El porcentaje de crecimiento a $35{ }^{\circ} \mathrm{C}$ se calculó considerando como $100 \%$ el crecimiento a $27{ }^{\circ} \mathrm{C}$.

\section{Tipo de compatibilidad}

El tipo de apareamiento se determinó cocultivando cada uno de los aislados monozoospóricos de $P$. capsici, con cada una de las cepas de tipo de compatibilidad conocida A1 y A2. Las confrontaciones se llevaron a cabo colocando en cajas Petri con agar V8C, fragmentos de micelio del aislado problema y de la cepa de tipo de compatibilidad conocida, con una separación de tres centímetros entre ellos. Las cajas fueron incubadas 2.5 semanas a $27^{\circ} \mathrm{C}$ en oscuridad. Se examinó al microscopio (objetivo 10X) la formación de oosporas. A los aislados que produjeron oosporas con la cepa A1, pero no con la A2 se determinaron como tipo de apareamiento A2 y a los que produjeron oosporas con A2 se determinaron como A1.

\section{Sensibilidad a mefenoxam}

Discos de agar de $8 \mathrm{~mm}$ de diámetro de los 31 cultivos monozoospóricos, fueron transferidos a cajas petri con medio V8C con y sin mefenoxam. Se empleó 100 ppm de mefenoxam (Ridomil Gold-EC, Syngenta, Greensboro, NC, 48\% ia.), agregado al medio previo al vaciado en cajas petri (Lamour y Hausbeck, 2000). Las placas fueron incubadas en oscuridad, a $25^{\circ} \mathrm{C}$ durante 7 días. Se evaluó cada $24 \mathrm{~h}$ el crecimiento radial del micelio, midiendo el diámetro en dos direcciones (perpendiculares) obteniendo el promedio.

El porcentaje de crecimiento relativo se calculó considerando como $100 \%$ el crecimiento promedio de cada aislado cultivado en medio sin mefenoxam (Lamour and Hausbeck. 2000; FernándezPavía, et al. 2004). Se efectuaron dos ensayos con tres repeticiones por aislado. Los aislados monozoospóricos fueron clasificados de acuerdo con la escala establecida por Lamour y Hausbeck (2000). Siendo sensibles (S) los que tuvieron un crecimiento relativo menor de $30 \%$, sensibilidad intermedia (SI) de 31-90\% e insensible (I) mayor al 90\% de crecimiento. 


\section{Análisis de virulencia}

Se realizaron tres repeticiones, con cada aislados, utilizando tres plantas/repetición en la etapa de tres a cuatro hojas verdaderas de la variedad Sonora Anaheim (Seminis). La inoculación se realizó colocando las plántulas en frascos estériles con $50 \mathrm{ml}$ de agua, a cada una se agregó una solución de $10^{4}$ zoosporas $\mathrm{ml}^{-1}$, más un antibiótico (ampicilina $100 \mathrm{mg} \mathrm{ml}^{-1}$ ) para evitar el crecimiento de bacterias.

Las plantas inoculadas, se colocaron en una cámara de crecimiento a $25^{\circ} \mathrm{C}$ y se evaluó el daño a los cinco días, usando la escala descrita por Parker y Grau (1992): 0= planta sana; $1=$ necrosis y obstrucción en la base del tallo; $2=$ necrosis, obstrucción en la base del tallo y marchitez parcial; $3=$ necrosis, obstrucción en la base del tallo, marchitez avanzada; 4= muerte total de la planta.

\section{Resultados y discusión}

\section{Patrón de crecimiento de la colonia}

En la población analizada se encontraron cinco patrones de crecimiento, siendo el tipo estrellado el más frecuente (62.5\%), seguido por el fillamentoso (15.62\%), estolonífero (12.5\%), toruloso $(6.25 \%)$ y petaloide $(3.12 \%)$ (Figura 1). Se observó una variación de los patrones de crecimiento en todo el estado, así como alta variabilidad dentro de algunos municipios, como Dolores Hidalgo en donde se encontraron tres tipos de crecimiento y Cortazar que en tres muestras tiene tres tipos de crecimiento.

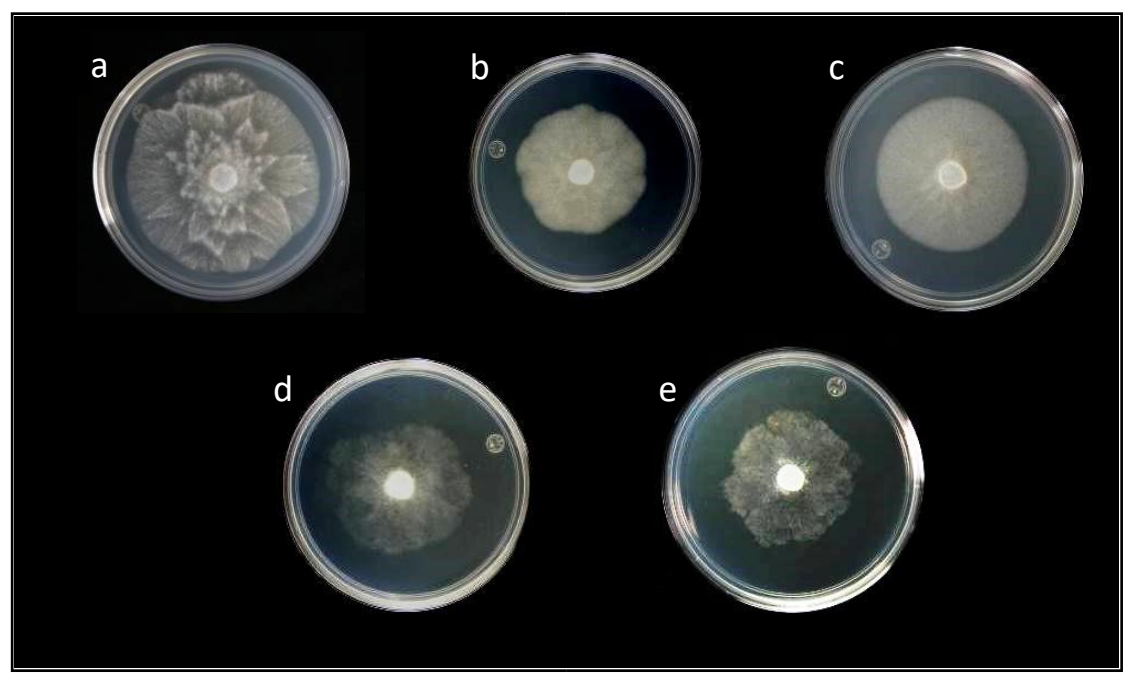

Figura 1. Morfotipos de colonias de $P$. capsici del estado de Guanajuato observadas en PDA. a) estrellado; b) filamentoso; c) estolonífera; d) toruloso; y e) petaloide.

En contraste con Silao y San Luis de la Paz que todos sus aislados muestran un tipo de crecimiento (Cuadro 1). Con un menor tamaño de muestra, se observó una mayor diversidad en formas de crecimiento en el estado de Guanajuato que en los aislados de Nuevo México, EE. UU. (FernándezPavía et al., 2004), Illinois, EE. UU. (Islam et al., 2005) y Chihuahua, México (Sánchez-Gurrola et al., 2019), coincidiendo con estos trabajos de que el crecimiento estrellado es el más frecuente. 
Cuadro 1. Características morfológicas y fisiológicas de 31 cultivos monoospóricos de Phytophthora capsici.

\begin{tabular}{|c|c|c|c|c|c|c|c|}
\hline Municipio & Clave & $\begin{array}{l}\text { Tipo de } \\
\text { chile }\end{array}$ & $\begin{array}{c}\text { Tipo de } \\
\text { crecimiento }\end{array}$ & $\begin{array}{c}\text { Tipo de } \\
\text { compatibilidad }\end{array}$ & $\begin{array}{c}\text { Crecimiento } \\
\text { a } 35^{\circ} \mathrm{C}^{1} \\
(\%)\end{array}$ & $\begin{array}{c}\text { Sensibilidad } \\
\text { mefenoxam }^{2} \\
(100 \text { ppm })\end{array}$ & $\begin{array}{c}\text { Grado de } \\
\text { virulencia }^{3}\end{array}$ \\
\hline \multirow{12}{*}{$\begin{array}{l}\text { Dolores } \\
\text { Hidalgo }\end{array}$} & D1 & Poblano & Estrellado & A1 & 15.16 & SI (68\%)a & 2 \\
\hline & D2 & Poblano & Estolonífero & $\mathrm{A} 2$ & 8.74 & SI $(43 \%) \mathrm{c}$ & 2 \\
\hline & D3 & Poblano & Estrellado & A1 & 17.48 & SI (63\%)ab & 4 \\
\hline & D4 & Poblano & Estrellado & $\mathrm{A} 2$ & 14.22 & SI $(55 \%) b$ & 1 \\
\hline & D5 & Poblano & Estolonífero & $\mathrm{A} 2$ & 11.91 & SI $(56 \%) b$ & 3 \\
\hline & D6 & Poblano & Estrellado & $\mathrm{A} 2$ & 11.86 & SI $(39 \%) c$ & 2 \\
\hline & D7 & Poblano & Estrellado & $\mathrm{A} 2$ & 11.41 & $\mathrm{~S}(27 \%) \mathrm{cd}$ & 2 \\
\hline & D8 & Poblano & Estolonífero & A1 & 11.25 & $\mathrm{~S}(25 \%) \mathrm{d}$ & 0 \\
\hline & D9 & Poblano & Toruloso & A1 & 12.49 & S (30\%)cd & 1 \\
\hline & D10 & Poblano & Toruloso & A1 & 0 & S (18\%)de & 1 \\
\hline & D11 & Poblano & Toruloso & A1 & 7.51 & SI $(44 \%) \mathrm{c}$ & 0 \\
\hline & D12 & Poblano & Toruloso & A1 & 5.95 & SI $(71 \%) a$ & 0 \\
\hline \multirow[t]{7}{*}{ Silao } & $\mathrm{S} 1$ & Güero & Estrellado & A1 & 19.62 & SI $(38 \%) c$ & 1 \\
\hline & $\mathrm{S} 2$ & Güero & Estrellado & A2 & 16.62 & $\mathrm{SI}(51 \%) \mathrm{bc}$ & 1 \\
\hline & S3 & Güero & Estrellado & A1 & 22.28 & SI $(48 \%) b c$ & 1 \\
\hline & $\mathrm{S} 4$ & Serrano & Estrellado & A1 & 3.21 & SI $(52 \%) b c$ & 1 \\
\hline & S5 & Jalapeño & Estrellado & A2 & 18.28 & $\mathrm{~S}(24 \%) \mathrm{d}$ & 1 \\
\hline & S6 & Jalapeño & Estrellado & $\mathrm{A} 2$ & 8.71 & $\mathrm{SI}(54 \%) \mathrm{b}$ & 1 \\
\hline & S7 & Jalapeño & Estrellado & A2 & 16.34 & $\mathrm{~S}(12 \%) \mathrm{e}$ & 1 \\
\hline Salvatierra & SA3 & Chilaca & Toruloso & A1 & 6.6 & SI $(36 \%) \mathrm{cd}$ & 2 \\
\hline \multirow{3}{*}{$\begin{array}{l}\text { San Luis } \\
\text { de la Paz }\end{array}$} & SP1 & Poblano & Estrellado & A1 & 18.29 & S (16\%)de & 2 \\
\hline & SP2 & Poblano & Estrellado & A1 & 4.23 & $\mathrm{~S}(22 \%) \mathrm{d}$ & 2 \\
\hline & SP3 & Poblano & Estrellado & A2 & 23.32 & S (15\%)de & 2 \\
\hline \multirow{3}{*}{$\begin{array}{c}\text { Juventino } \\
\text { Rosas }\end{array}$} & $\mathrm{J} 1$ & Jalapeño & Filamentoso & A1 & 8.3 & SI $(39 \%) c$ & 3 \\
\hline & $\mathrm{J} 2$ & Poblano & Estrellado & A1 & 15.32 & S (18\%)de & 2 \\
\hline & $\mathrm{J} 3$ & Jalapeño & Filamentoso & A1 & 7.86 & SI $(47 \%) b c$ & 2 \\
\hline \multirow[t]{3}{*}{ Cortázar } & CT1 & Jalapeño & Estolonífero & A1 & 28.37 & S $(28 \%) \mathrm{cd}$ & 1 \\
\hline & CT3 & Jalapeño & Petaloide & A1 & 12.57 & SI $(40 \%) \mathrm{c}$ & 2 \\
\hline & CT4 & Jalapeño & Estrellado & A1 & 30.67 & SI $(59 \%) a b$ & 2 \\
\hline \multirow{2}{*}{$\begin{array}{l}\text { Cepas de } \\
\text { referencia }\end{array}$} & $\mathrm{PCC}$ & SD & Estolonífero & $\mathrm{A} 2$ & 23.21 & SI $(54 \%) b$ & 1 \\
\hline & PCT & SD & Estolonífero & A1 & 11.36 & SI $(55 \%) b$ & 1 \\
\hline
\end{tabular}

${ }^{1}=$ porcentaje de crecimiento considerando $100 \%$ el crecimiento a $27{ }^{\circ} \mathrm{C} ;{ }^{2}=$ sensibilidad a mefenoxam; $\mathrm{S}=$ sensible; SI = sensibilidad intermedia; $\mathrm{I}=$ insensible; ${ }^{3}=$ escala; $0=$ sin síntomas; $1=$ daño en tallo; $2=$ marchitez parcial y clorosis; $3=$ marchitez avanzada; $4=$ planta muerta. 
Al igual que en los trabajos mencionados, en este estudio, no se encontró una relación clara del tipo de crecimiento con las otras características evaluadas. La variación en la morfología de la colonia se ha observado en $P$. capsici desde hace varios años y es considerada un primer indicio de la diversidad existente (Bowers et al., 2007).

Crecimiento a $35{ }^{\circ} \mathrm{C}$. La tasa de crecimiento promedio a $27{ }^{\circ} \mathrm{C}$ de los cultivos monozoospóricos fue de $12.82 \mathrm{~mm} \mathrm{día}^{-1}$, este crecimiento fue mayor a lo reportado por Tamietti y Valentino (2001), quienes encontraron para 20 aislados de $P$. capsici una tasa promedio de crecimiento de $0.81 \mathrm{~mm}$ día $^{-1}$ y a la reportada por Fernández-Pavía et al. (2004) de $9 \mathrm{~mm} \mathrm{día}^{-1}$ para aislados de Nuevo México crecidos a $30^{\circ} \mathrm{C}$.

La tasa de crecimiento promedio de los cultivos monozoospóricos a $35^{\circ} \mathrm{C}$ fue de $1.7 \mathrm{~mm} \mathrm{día}^{-1}$ variando entre 0 y $3.87 \mathrm{~mm}^{\text {día }}{ }^{-1}$ (dato no mostrado); este resultado es menor al obtenido por Islam et al. (2005), quienes observaron que la tasa de crecimiento a esta temperatura para aislados de Illinois varió de 3.1 a $10.1 \mathrm{~mm} \mathrm{día}^{-1}$ y a lo reportado por Granke et al. (2011) quienes encontraron un crecimiento promedio de $3.4 \mathrm{~mm}_{\text {día }}{ }^{-1}$ en 122 aislados de $P$. capsici originarios de 12 países. El porcentaje de crecimiento de los aislados de Guanajuato a $35^{\circ} \mathrm{C}$ varió de 0 a $30.7 \%$, todos los aislados con excepción del CT4, tuvieron un crecimiento menor al 30\% (Cuadro 1).

Este resultado es contrastante al obtenido para aislados de Nuevo México cuyo crecimiento varió entre 11 y 100\% (Fernández-Pavía et al., 2004). Pero concuerda con lo reportado por TlalpalBolaños et al. (1995) quienes reportan que los aislados de México crecen pobremente a esta temperatura.

Este crecimiento sugiere que los aislados de Guanajuato presentan una menor adaptación a temperaturas elevadas, esto puede ser debido a las condiciones térmicas en las que han evolucionado ya que de acuerdo con el INEGI (2013), históricamente la temperatura media anual en Guanajuato es de $18{ }^{\circ} \mathrm{C}$ y la temperatura promedio más alta es alrededor de $30{ }^{\circ} \mathrm{C}$ y se presenta en los meses de mayo y junio cuando el ciclo de cultivo para chile en fresco está terminando en este estado.

\section{Tipo de compatibilidad}

Todos los aislados fueron heterotálicos y la distribución de los tipos de compatibilidad en el estado fue al azar y en algunos campos se encontraron conviviendo los dos tipos de compatibilidad, observándose en el estado una predominancia del tipo A1 (65.5\%) (Cuadro 1).

A nivel mundial, la identificación de ambos tipos de compatibilidad en colectas de $P$. capsici se ha reportado frecuentemente por diferentes investigadores, como Fernández-Pavía et al. (2004), los cuales al caracterizar 59 aislados del sur de Nuevo México, EE. UU., encontraron una distribución al azar de ambos tipos de compatibilidad A1 y A2 y demostraron la convivencia de ellos en un solo campo, señalando al A1 como el de mayor frecuencia en una relación de 2:1 con respecto al A2. Tamietti y Valentino (2001), en una colecta de 26 aislados originarios del norte de Italia, encontraron 19 del tipo A1, 3 del tipo A2 y 4 heterotálicos. Islam et al. (2005) reportan en una colecta de 57 aislados realizada en Illinois E.U., 31 del tipo A1 y 26 del tipo A2. Meitz et al. (2010) encontraron en una colección de 78 aislados de Sudáfrica 42 del tipo A1 y 36 del tipo A2. 
En México, Castro-Rocha et al. (2016) analizaron 81 aislados de $P$. capsici de cuatro estados, incluyendo 14 aislados de Guanajuato. Informan la presencia de 32 aislados con el tipo de apareamiento A1, 43 con el tipo A2 y 6 homotálicos en el total de la población. También se ha confirmado la presencia de ambos tipos de compatibilidad en zonas productoras de chile en los estados de Aguascalientes, Chihuahua, Ciudad de México, Jalisco, Michoacán y Zacatecas (Rodríguez-Moreno et al., 2004; Silva-Rojas et al., 2009; Castro-Rocha et al., 2016).

La presencia de los dos tipos de compatibilidad en Guanajuato fue reportada por Pérez-Moreno et al. (2003), quienes al analizar 8 aislados obtenidos en dos municipios de este estado, reportan 4 del tipo A1 y 4 del tipo A2, esto fue confirmado por el trabajo de Castro-Rocha et al. (2016) que reportan haber encontrado en Guanajuato 5 aislados del tipo A1, 6 del tipo A2 y 3 homotálicos.

Coincidiendo con los resultados del presente trabajo, ambos tipos de apareamiento se han identificado dentro de la misma parcela por varios investigadores (Gobena et al., 2012a; Jiang et al., 2015; Barchenger et al., 2017). Esto indica que el manejo de la enfermedad será más difícil, debido a la diversidad genética generada por reproducción sexual, que puede originar nuevos genotipos resistentes a tratamientos químicos o con mayor capacidad patogénica o virulencia y aislados capaces de romper la resistencia de variedades en menor tiempo.

\section{Sensibilidad a mefenoxam}

El $65.5 \%$ de los aislados presentaron sensibilidad intermedia a mefenoxam a 100 ppm, siendo el resto sensibles (Cuadro 1). El crecimiento del micelio de los aislados con sensibilidad intermedia fue malformado, con hifas cortas y en algunos casos turulosas. La insensibilidad de los aislados de $P$. capsici a este compuesto químico se ha reportado desde hace varios años, se ha señalado que se debe a que los oomicetos, aunque morfológicamente similares a los hongos verdaderos, son genética y bioquímicamente diferentes (Erwin y Ribeiro, 1996) y no son susceptibles a la mayoría de los fungicidas de amplio espectro, por esta razón, los productores tienden a depender de un número limitado de fungicidas.

Los más populares a nivel mundial y de los que se ha abusado en su uso, son los fungicidas de la clase de las fenilamida, específicamente metalaxil y su enantiómero mefenoxam (Ridomil Gold EC) (Lamour y Hausbeck, 2000), de los cuales se ha establecido que su modo de acción es sitio específico y que la tolerancia a ellos está condicionada por un locus único que tiene un efecto mayor y dominancia incompleta, sujeto a modificación por genes de efectos menores (Fabritius et al., 1997).

Además, se ha reportado que la insensibilidad al mefenoxam, también confiere insensibilidad al metalaxil, en poblaciones de campo de P. capsici en pimiento morrón (Parra y Ristaino, 1998). En Guanajuato, el mefenoxam y metalaxil se han utilizado por más de una década en el cultivo de chile, por lo que la aparición de aislados con insensibilidad a estos productos era de esperarse.

Aislados con insensibilidad al metalaxil fueron reportados por primera vez en Guanajuato por Pérez-Moreno et al. (2003), quienes señalan haber encontrado nueve aislamientos insensibles a metalaxil. Estos resultados y los encontrados en este trabajo, sugieren que en el estado de Guanajuato no se han encontrado aún aislados insensibles a mefenoxam, hay una tendencia a desarrollar resistencia, lo que podría ser un problema a corto plazo. 
Dado que, de acuerdo con estudios realizados por Lamour y Hausbeck (2001), una vez establecida la resistencia a mefenoxam o metalaxil, el uso de estos debe ser limitado, pues la frecuencia de aislados resistentes no decrece durante dos años, aun eliminando la aplicación del químico y empleando rotación de cultivo.

\section{Análisis de virulencia}

Dos de los aislados más virulentos D3 y D5 y los tres aislados no virulentos D8, D11 y D12 encontrados en Guanajuato, son originarios del municipio de Dolores Hidalgo, lo que refleja una alta variabilidad en esta característica, esto podría atribuirse a la presencia de los dos tipos de apareamiento en este municipio, lo cual sugeriría la existencia de reproducción sexual y por tanto la aparición de nuevas razas patogénicas, pero al analizar otros municipios, encontramos localidades como Silao en donde se encontraron también los dos tipos de apareamiento, pero solo un nivel de virulencia en sus aislados y el otro caso es el de Juventino Rosas y Cortazar en donde se tiene un solo tipo de compatibilidad y variación en el grado de virulencia (Cuadro 1).

La falta de correlación de la virulencia con otras características como el tipo de apareamiento, el crecimiento radial a 27 y a $35^{\circ} \mathrm{C}$, concuerda con los estudios reportado por Fernández-Pavía et al. (2004) y Tamietti y Valentino (2001). La alta diversidad en la virulencia presente en el municipio de Dolores Hidalgo, podría estar relacionada con el tipo de hospedero, ya que si bien, es un mismo tipo de chile el que se siembra, todos ellos son variedades criollas y prácticamente cada productor tiene su propia variedad y produce su semilla lo que genera un proceso de selección.

Esto podría haber originado que cada criollo desarrollara diferentes mecanismos de defensa para evitar o limitar el daño producido por Phytophthora. Así, los patógenos tendrían que evolucionar para generar nuevos mecanismos de virulencia que les permita eludir las defensas de los huéspedes. Provocando que los huéspedes, tengan que evolucionar para generar nuevas defensas para enfrentar a los nuevos mecanismos de virulencia de los patógenos. Estas presiones de selección recíprocas entre los mecanismos de virulencia del patógeno y de defensa de la planta, se vería reflejado en la diversidad en la virulencia que se está encontrando. Esto habría que probarlo con otros estudios ya que no fue el objetivo de este trabajo.

La población de Phytophthora capsici de Guanajuato se caracteriza por sus altos niveles de diversidad, similares a lo reportado por varios autores para otras poblaciones de este patógeno (Lamour y Hausbeck, 2001; Hausbeck y Lamour, 2004; Gevens et al., 2008; Meitz et al., 2010; Lamour et al., 2011; Gobena et al., 2012b), por lo que esta diversidad debe de ser considerada para el diseño de estrategias para su control.

\section{Conclusiones}

Existe variabilidad fenotípica entre los aislados de $P$. capsici de Guanajuato. En Guanajuato se encuentran los dos tipos de apareamiento, siendo el A1 el predominante. Existen municipios en donde conviven aislados de los dos tipos de compatibilidad.

Una alta proporción de aislados de $P$. capsici han desarrollado resistencia intermedia a Mefenoxam. Existe una alta diversidad en la virulencia presente en el municipio de Dolores Hidalgo. 
No se observa una relación directa entre la forma de crecimiento, el crecimiento a $35^{\circ} \mathrm{C}$, el tipo de compatibilidad, la resistencia a mefenoxam, y el grado de virulencia.

\section{Agradecimientos}

Los resultados son parte del proyecto fiscal: 'desarrollo y transferencia de tecnologías sustentables para la producción de chile y jitomate en campo e invernadero’. No. 167834791.

\section{Literatura citada}

Barchenger, D. W.; Lamour, K. H.; Zong-Ming, S.; Shrestha, S.; Kumar, S.; Shih-Wen, L.; Burlakoti, R. and Bosland, P. W. 2017. Intra- and intergenomic variation of ploidy and clonality characterize Phytophthora capsici on Capsicum sp. in Taiwan. Mycol. Prog. 16(10):955-963. doi: 10.1007/s11557-017-1330-0.

Bowers, J. H.; Martin, F. N.; Tooley, P. W. and Luz, E. D. M. N. 2007. Genetic and morphological diversity of temperate and tropical isolates of Phytophthora capsici. Phytopathology. 97(4):492-503. http://dx.doi.org/10.1094/PHYTO-97-4-0492

Castro-Rocha, A.; Shrestha, S.; Lyon, B.; Grimaldo-Pantoja, G. L.; Flores-Marges, J. P.; ValeroGalván, J.; Aguirre-Ramírez, M.; Osuna-Ávila, P.; Gómez-Dorantes, N.; Ávila-Quezada, G.; Luna-Ruíz, J. J.; Rodríguez-Alvarado, G.; Fernández-Pavía, S. P. and Lamour, K. 2016. An initial assessment of genetic diversity for Phytophthora capsici in northern and central Mexico. Mycol Progress. 15(1):1-12. Doi 10.1007/s11557-016-1157-0.

Erwin, D. C. and Ribeiro, O. K. 1996. Phytophthora diseases worldwide. APS PRESS. The American Phytopathological Society. St. Paul, Minnesota. 562 p.

Fabritius, A.; Shattock, R. C. and Judelson, H. S. 1997. Genetic analysis of metalaxyl insensitivity loci in Phytophthora infestans using linked DNA markers. Phytopathology. 87(10):10341040. http://dx.doi.org/10.1094/PHYTO.1997.87.10.1034.

Fernández-Pavía, S. P.; Biles, C. L.; Waugh, M. E.; Onsurez-Waugh, K.; Rodríguez-Alvarado, G. and Liddell, C. M. 2004. Characterization of Southern New Mexico Phytophthora capsici Leonian isolates from pepper (Capsicum annuum L.). Rev. Mex. Fitopatol. 22(1):82-89.

Gevens, A. J.; Donahoo, R. S.; Lamour, K. H. and Hausbeck, M. K. 2008. Characterization of Phytophthora capsici causing foliar and pod blight of snap bean in Michigan. Plant Dis. 92(2):201-209.

Gobena, D.; Garth, M. T. and Lamour, K. H. 2012a. Survival and spread of Phytophthora capsici on Long Island, New York. Mycol. Prog. 11(3):761-768. https://doi.org/10.1007/s11557011-0787-5.

Gobena, D.; Roig, J.; Galmarini, C.; Hulvey, J. and Lamour, K. H. 2012b. Genetic diversity of Phytophthora capsici isolates from pepper and pumpkin in Argentina. Mycologia. 104(1):102-107.

Granke, L. L.; Quesada-Ocampo, L. M. and Hausbeck, M. K. 2011. Variation in phenotypic characteristics of Phytophthora capsici isolates from a worldwide collection. Plant Dis. 95(9):1080-1088.

INEGI. 2013. Instituto Nacional de Estadística y Geografía. Conociendo Guanajuato. Aguascalientes, Aguascalientes. México. Serie Conociendo México. Folleto informativo. $30 \mathrm{p}$. 
Islam, S. Z.; Babadoost, M.; Lambert, K. N. and Ndeme, A. 2005. Characterization of Phytophthora capsici isolates from processing pumpkin in Illinois. Plant Dis. 89(2):191-197.

Hausbeck, M. K. and Lamour, K. H. 2004. Phytophthora capsici on vegetable crops: research progress and management challenges. Plant Dis. 88(11):1292-1303.

Jiang, L.; Sanogo, S. and Bosland, P. W. 2015. Using recombinant inbred lines to monitor changes in the race structure of Phytophthora capsici in chili pepper in New Mexico. Plant Health Prog. 16(4):235-240. doi: 10.1094/PHP-RS-15-0034.

Lamour, K. H. and Hausbeck, M. K. 2000. Mefenoxam insensitivity and the sexual stage of Phytophthora capsici in Michigan cucurbit fields. Phytopathology. 90(4):396-400.

Lamour, K. H. and Hausbeck, M. K. 2001. The dynamics of mefenoxam insensitivity in a recombining population of Phytophthora capsici characterized with amplified fragment length polymorphism markers. Phytopathology. 91(6):553-557.

Lamour, K. H. and Hausbeck, M. K. 2003. Susceptibility of mefenoxam-treated curcubits to isolates of Phytophthora capsici sensitive and insensitive to mefenoxam. Plant Dis. 87(8):920-922. https://doi.org/10.1094/pdis.2003.87.8.920.

Lamour, K. H.; Stam, R.; Jupe, J. and Huitema, E. 2011. The oomycete broad-host-range pathogen Phytophthora capsici. Mol Plant Pathol. 13(4):329-337. Doi: 10.1111/j.13643703.2011.00754.x.

Martin, F. N.; Abad, Z. G.; Balci, Y. and Ivors, K. 2012. Identification and detection of Phytophthora: reviewing our progress, identifying our needs. Plant Dis. 96(8):1080-1103.

Meitz, J. C.; Linde, C. C.; Thompson, A.; Langenhoven, S. and Leod, A. 2010. Phytophthora capsici on vegetable hosts in South Africa: distribution, host range and genetic diversity. Austr. Plant Path. 39(5):431-439.

McDonald, B. A. and Linde C. 2002. Pathogen population genetics, evolutionary potential, and durable resistance. Annu Rev Phytopathol. 40(1):349-379. doi: 10.1146/annurev.phyto.40.120501.101443. Epub 2002 Feb 20. PMID: 12147764.

Parker, J. L. and Grau, C. R. 1992. Aphanomyces. In: 'Methods for research on soilborne phytopathogenic fungi'. (Ed.). Singleton, L. L.; Mihail, J. D. and Rush, C. M. APS Press, USA. 27-30 pp.

Parra, G. and Ristaino, J. B. 1998. Insensitivity to Ridomil Gold (mefenoxam) found among field isolates of Phytophthora capsici causing Phytophthora blight on bell pepper in North Carolina and New Jersey. Plant Dis. 82(6):711-711. http://dx.doi.org/10.1094/ PDIS.1998.82.6.711D.

Pérez-Moreno, L.; Durán-Ortiz, L.; Ramírez-Malagón, R.; Sánchez-Palé, R. y Olalde-Portugal, V. 2003. Compatibilidad fisiológica y sensibilidad a fungicidas de aislamientos de Phytophthora capsici Leo. Rev. Mex. Fitopatol. 21(1):19-25.

Rodríguez-Moreno, V. M.; Luna-Ruiz, J. J.; Valle-García, P.; Tiscareño-López, M. y Ruiz-Corral, J. A. 2004. Caracterización patogénica y sexual de Phytophthora capsici Leonian y análisis de su distribución espacial en el Centro-Norte de México mediante un sistema de información geográfica. Rev. Mex. Fitopatol. 22(1):72-81.

Sánchez-Gurrola, C.; Gómez-Dorantes, N.; Rodríguez-Alvarado, G.; Fernández-Pavía, S. P. y Ávila-Quezada, G. 2019. Variabilidad morfológica y sensibilidad de Phytophthora capsici causando marchitez en chile pimiento morrón en Chihuahua, México. Rev. Mex. Fitopatol. 37(1):65-71. Doi: 10.18781/R.MEX.FIT.1904-4. 
Silva-Rojas, H. V.; Fernández-Pavía, S. P.; Góngora-Canul, C.; Macías-López, B. C. y ÁvilaQuezada, G. D. 2009. Distribución espacio temporal de la marchitez del chile (Capsicum annuum L.) en Chihuahua e identificación del agente causal Phytophthora capsici Leo. Revista Mexicana de Fitopatología 27(2):134-147.

Tamietti, G. and Valentino, N. 2001. Physiological characterization of a population of Phytophthora capsici Leon., from northern Italy. J. Plant Pathol. 83(3):199-205.

Tlalpal-Bolaños, B.; Osada-Kawasoe, S.; González-Cossio, F. y Mendoza-Zamora, C. 1995. Comportamiento fisiológico de 30 aislamientos de Phytophthora capsici Leo. Rev. Mex. Fitopatol. 13(1):41-51. 